\title{
DIPHYES BLUME, A FORGOTTEN GENUS OF THE SUBTRIBE BULBOPHYLLINAE (ORCHIDACEAE, EPIDENDROIDEAE)
}

\author{
DARIUSZ L. SZlACHETKO*, PIOTR RUTKOWSKI \\ Departemnt of Plant Taxonomy and Nature Conservation, Gdansk University \\ Al. Legionów 9, 80-441 Gdańsk, Poland \\ * e-mail: biodarek@univ.gda.pl
}

(Received: March 20, 2007. Accepted: October 4, 2007)

ABSTRACT

The genus Diphyes Blume is reinstated. 62 new combinations on the species level are validated.

KEY WORDS: Orchidaceae, Epidendroideae, Bulbophyllinae, Bulbophyllum, Diphyes, paleotropics.

Carl Ludwig von Blume (1796-1862) was one of the most prolific botanist of his time. He described many new genera and species mostly from SE Asia. After years of oblivion his taxa are reapproved. Such genera as Echioglossum, Crepidium or Osyricera to mention a few, are assigned to Blume. The genus Diphyes was described by Blume in 1825 in his fundamental work about orchids of the former Dutch East Indies. The genus was divided into 8 sections: Monanthes Bl. (with the only species $D$. tortuosa B1.), Diptychanthes B1. (D. mutabilis B1.), Stachysanthes B1. (D. flavescens B1., D. gibbosa B1., D. odorata B1.), Desmosanthes B1. (D. angustifolia B1., D. capitata B1., D. crocea B1., D. obtusa B1., D. sulcata B1.), Corymbosia B1. (D. laxiflora B1.), Polymeres B1. (D. mucronata B1., D. tenuifolia B1., D. violacea B1.), Diasperia B1. (D. cernua B1., D. ciliata B1., D. pusilla B1.) and Macrocaulia B1. (D. gracilis B1., D. hirsuta B1., D. inaequalis B1., D. ovalifolia B1., D. tenella $\mathrm{B} 1$.). As thus can be seen, Blume did not recognize typical section, what was a normal procedure at that time. Unfortunately, it causes that the lectotypification of Diphyes is difficult. From among Blume's sections, only Desmosanthes was transferred to Bulbophyllum, all other have been ignored or reduced to the synonymy.

Detailed examination of the original description of the genus and comparing it with species included by Blume to Diphyes led us to the conclusion that the genus should be typified by $D$. tortuosa. It is the only species of Blume's content of Diphyes with ,sepalia...basi leviter cohaerentia...". In any other species originally attributed to $D i$ phyes the sepals are free to the base (Table 1).

\section{Diphyes Blume}

Bijdr. Fl. Ned. Ind.: 310, t. 66. 1825; LECTOTYPE (hoc loco designatus): Diphyes tortuosa $\mathrm{Bl}$.

Incl.: Bulbophyllum Thouars sect. Polyblepharon Schltr., Repert. Sp. Nov. Regni Veg., Beih. 1:P 701 \& 794. 1913.
Plants usually small, with pending rhizome. Pseudobulbs narrowly cylindrical, enveloped basally by sheaths. Leaf single. Inflorescence single-flowered, much shorter than the pseudobulbs. Flowers small, non-resupinate. Sepals much larger than petals. Lateral sepals connate near the base or almost up to apices forming a boat-shaped structure, inrolled or flat apically. Lip small, oblong-ovate to linear, more or less ciliate along margins. Column foot nearly as long as the column part. Stelidia prominent, digitate, longer than anther.

The genus is widely distributed from Bhutan to Australia, with centre of diversification in New Guinea. It comprises over 60 species.

Diphyes appears to be somewhat similar to the genus Rhytionanthos Garay et al. by having horn-like structure formed by the fusion of lateral sepals. In Diphyes, however, the horn is open on the upper surface with bi-lobed apex, whereas in Rhytionanthos it is closed at the bottom and upper surface and lateral sepals are connate to the apex. Pelma Finet is another genus resembling Diphyes in habit and in general flower structure. In Pelma the lip is concave at the base, and the cavity is partly covered by two converging teeth whereas in Diphyes is ellipsoid, and almost flat on the upper surface. In all other respects, however, Diphyes seems to occupy rather an isolated position in the subtribe Bublophyllinae.

The following species belong to the genus:

Diphyes aberrans (Schltr.) Szlach. \& Rutk., comb. nov. Basionym: Bulbophyllum aberrans Schltr., Repert. Spec. Nov. Regni Veg. 10: 177. 1911.

Diphyes adenoblepharon (Schltr.) Szlach. \& Rutk., comb. nov.

Basionym: Bulbophyllum adenoblepharon Schltr., Repert. Spec. Nov. Regni Veg., Beih. 1: 803. 1913. 
TABLE 1. Taxonomic position of Diphyes's species' according to the various authors.

\begin{tabular}{cc}
\hline Taxa described & Current status \\
by Blume (1825) & (cf. Comber 1990, Seidenfaden 1979, \\
D. = Diphyes & Seidefaden \& Wood 1992, Vermeulen 1991) \\
& B. = Bulbophyllum \\
\hline
\end{tabular}

Diptychanthes B1. =Aphanobulbon Schltr.

D. mutabilis $\mathrm{Bl} . \quad=$ B. mutabile $(\mathrm{Bl}$.) Lindl.

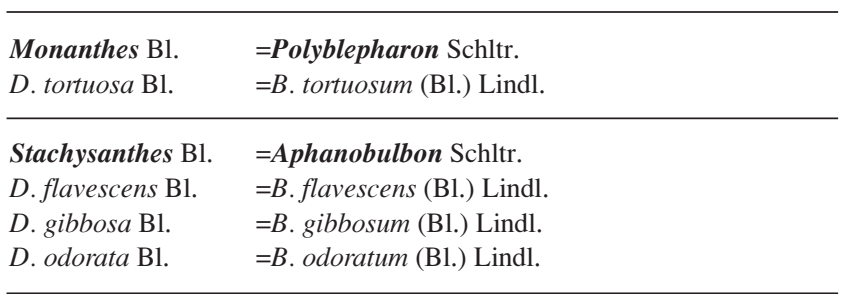

\begin{tabular}{|c|c|}
\hline Desmosanthes B1. & $=$ Desmosanthes $(\mathrm{Bl}$.) J.J.Smith \\
\hline D. angustifolia $\mathrm{B} 1$. & $=B$. angustifolium (B1.) Lindl. \\
\hline D. capitata $\mathrm{Bl}$. & $=B$. capitatum $(\mathrm{B} 1$.) Lindl. \\
\hline D. crocea $\mathrm{Bl}$. & $=$ B. croceum $(\mathrm{Bl}$.) Lindl. \\
\hline D. obtusa B1. & $=$ B. obtusum (B1.) Lindl. \\
\hline D. sulcata $\mathrm{B} 1$. & $=$ B. sulcatum $(\mathrm{Bl}$.) Lindl. \\
\hline Corymbosia Bl. & $=$ Desmosanthes (B1.) J.J.Sm. \\
\hline D. laxiflora Bl. & = B.laxiflorum (B1.) Lindl. \\
\hline Polymeres B1. & $=$ Micromonanthe or Megaloglossum \\
\hline D. mucronata $\mathrm{Bl}$. & $\begin{array}{l}=\text { B. mucronatum (B1.) Lindl. (Micromonanthe } \\
\text { Schltr.) }\end{array}$ \\
\hline D. tenuifolia $\mathrm{Bl}$. & $\begin{array}{l}=\text { B. tenuifolium (B1.) Lindl. (Micromonanthe Schltr } \\
\text { or Megaglossum Carr) }\end{array}$ \\
\hline D. violacea $\mathrm{B} 1$. & $=$ B. violaceum $(\mathrm{Bl}$.$) Lindl. ($ Megaglossum Carr $)$ \\
\hline Diasperia $\mathrm{Bl}$. & =Monilibulbus J.J.Smith \\
\hline D. cernua $\mathrm{B} 1$. & $=B$. cernuum $(\mathrm{B} 1$.) Lindl. \\
\hline D. ciliata $\mathrm{Bl}$. & $=$ B. ciliatum $(\mathrm{Bl}$.) Lindl. (Micromonanthe Schltr.) \\
\hline D. pusilla $\mathrm{Bl}$. & $=B$. ovalifolium $(\mathrm{Bl}$.$) Lindl.$ \\
\hline Macrocaulia $\mathrm{Bl}$. & $=$ Monilibulbus J.J.Smith \\
\hline D. gracilis $\mathrm{B} 1$. & $=$ B. schefferi (O.Ktze.) Schltr. \\
\hline D. hirsuta $\mathrm{Bl}$. & $=$ B. ovalifolium $(\mathrm{B} 1$.$) Lindl.$ \\
\hline D. inaequalis $\mathrm{Bl}$. & $\begin{array}{l}=B \text {. inaequale }(\mathrm{Bl} \text {.) Lindl. } \\
\text { or } B \text {. ovalifolium (B1.) Lindl. }\end{array}$ \\
\hline D. ovalifolia Bl. & $=$ B. ovalifolium $(\mathrm{B} 1$.$) Lindl.$ \\
\hline D. tenella $\mathrm{B} 1$. & $=$ B. tenellum $(\mathrm{Bl}$.) Lindl. \\
\hline
\end{tabular}

Diphyes amblyanthum (Schltr.) Szlach. \& Rutk., comb. nov.

Basionym: Bulbophyllum amblyanthum Schltr., Repert. Spec. Nov. Regni Veg., Beih. 1: 814. 1913.

Diphyes angusteovatum (Seidenf.) Szlach. \& Rutk., comb. nov.

Basionym: Bulbophyllum angusteovatum Seidenf., Dansk Bot. Ark. 33(3): 41. 1979.

Diphyes arachnoideum (Schltr.) Szlach. \& Rutk., comb. nov.

Basionym: Bulbophyllum arachnoideum Schltr., Repert. Spec. Nov. Regni Veg., Beih. 1: 798. 1913.

Diphyes bicaudatum (Schltr.) Szlach. \& Rutk., comb. nov. Basionym: Bulbophyllum bicaudatum Schltr., Repert. Spec. Nov. Regni Veg., Beih. 1: 815. 1913.
Diphyes bisepalum (Schltr.) Szlach. \& Rutk., comb. nov. Basionym: Bulbophyllum bisepalum Schltr. In: K.Schum. and Lauterb., Nachtr. Fl. Deutsch. Sudsee: 196. 1905.

Diphyes blepharicardium (Schltr.) Szlach. \& Rutk., comb. nov.

Basionym: Bulbophyllum blepharicardium Schltr., Repert. Spec. Nov. Regni Veg., Beih. 1: 812. 1913.

Diphyes blephariglossum (Schltr.) Szlach. \& Rutk., comb. nov.

Basionym: Bulbophyllum blephariglossum Schltr., Repert Spec. Nov. Regni Veg., Beih. 1: 798. 1913.

Diphyes cadetioides (Schltr.) Szlach. \& Rutk., comb. nov. Basionym: Bulbophyllum cadetioides Schltr., Repert. Spec. Nov. Regni Veg., Beih. 1: 804. 1913.

Diphyes ciliolatum (Schltr.) Szlach. \& Rutk., comb. nov. Basionym: Bulbophyllum ciliolatum Schltr., Repert. Spec. Nov. Regni Veg., Beih. 1: 809. 1913.

Diphyes cryptanthum (Schltr.) Szlach. \& Rutk., comb. nov.

Basionym: Bulbophyllum cryptanthum Schltr. in K.Schum. \& Lauterb., Nachtr. Fl. Deutsch. Sudsee 199. 1905.

Diphyes decumbens (Schltr.) Szlach. \& Rutk., comb. nov. Basionym: Bulbophyllum decumbens Schltr., Repert. Spec. Nov. Regni Veg., Beih. 1: 820. 1913.

Diphyes dichaeoides (Schltr.) Szlach. \& Rutk., comb. nov. Basionym: Bulbophyllum dichaeoides Schltr., Repert. Spec. Nov. Regni Veg.. Beih. 1: 802. 1913.

Diphyes dichilus (Schltr.) Szlach. \& Rutk., comb. nov. Basionym: Bulbophyllum dichilus Schltr., Repert. Spec. Nov. Regni Veg., Beih. 1: 813. 1913.

Diphyes dryadum (Schltr.) Szlach. \& Rutk., comb. nov. Basionym: Bulbophyllum dryadum Schltr., Repert. Spec. Nov. Regni Veg., Beih. 1: 802. 1913.

Diphyes eciliatum (Schltr.) Szlach. \& Rutk., comb. nov. Basionym: Bulbophyllum eciliatum Schltr., Repert. Spec. Nov. Regni Veg., Beih. 1: 814. 1913.

Diphyes elegantius (Schltr.) Szlach. \& Rutk., comb. nov. Basionym: Bulbophyllum elegantius Schltr., Repert. Spec. Nov. Regni Veg., Beih. 1: 800. 1913.

Diphyes endotrachys (Schltr.) Szlach. \& Rutk., comb. nov. Basionym: Bulbophyllum endotrachys Schltr., Repert. Spec. Nov. Regni Veg., Beih. 1: 808. 1913.

Diphyes finisterrae (Schltr.) Szlach. \& Rutk., comb. nov. Basionym: Bulbophyllum finisterrae Schltr., Repert. Spec. Nov. Regni Veg., Beih. 1: 818. 1913.

Diphyes fuscatum (Schltr.) Szlach. \& Rutk., comb. nov. Basionym: Bulbophyllum fuscatum Schltr., Repert. Spec. Nov. Regni Veg., Beih. 1: 810. 1913. 
Diphyes glabrum (Schltr.) Szlach. \& Rutk., comb. nov. Basionym: Bulbophyllum glabrum Schltr., Repert. Spec. Nov. Regni Veg., Beih. 1: 822. 1913.

Diphyes heteroblepharon (Schltr.) Szlach. \& Rutk., comb. nov.

Basionym: Bulbophyllum heteroblepharon Schltr., Repert. Spec. Nov. Regni Veg., Beih. 1: 807. 1913.

Diphyes inauditum (Schltr.) Szlach. \& Rutk., comb. nov. Basionym: Bulbophyllum inauditum Schltr., Repert. Spec. Nov. Regni Veg., Beih. 1: 815. 1913.

Diphyes incumbens (Schltr.) Szlach. \& Rutk., comb. nov. Basionym: Bulbophyllum incumbens Schltr., Repert. Spec. Nov. Regni Veg., Beih. 1: 815. 1913.

Diphyes inversum (Schltr.) Szlach. \& Rutk., comb. nov. Basionym: Bulbophyllum inversum Schltr., Repert. Spec. Nov. Regni Veg., Beih. 1: 806. 1913.

Diphyes kenejianum (Schltr.) Szlach. \& Rutk., comb. nov. Basionym: Bulbophyllum kenejianum Schltr., Repert. Spec. Nov. Regni Veg., Beih. 1: 800. 1913.

Diphyes lichenoides (Schltr.) Szlach. \& Rutk., comb. nov. Basionym: Bulbophyllum lichenoides Schltr., Repert. Spec. Nov. Regni Veg., Beih. 1: 808. 1913.

Diphyes loxophyllum (Schltr.) Szlach. \& Rutk., comb. nov. Basionym: Bulbophyllum loxophyllum Schltr., Repert. Spec. Nov. Regni Veg., Beih. 1: 817. 1913.

Diphyes maboroense (Schltr.) Szlach. \& Rutk., comb. nov. Basionym: Bulbophyllum maboroense Schltr., Repert. Spec. Nov. Regni Veg., Beih. 1: 809. 1913.

Diphyes membranaceum (Schltr.) Szlach. \& Rutk., comb. nov.

Basionym: Bulbophyllum membranaceum Teijsm. \& Binn., Ned. Kruidk. Arch. 3: 397. 1855.

Diphyes microblepharon (Schltr.) Szlach. \& Rutk., comb. nov.

Basionym: Bulbophyllum microblepharon Schltr., Repert. Spec. Nov. Regni Veg., Beih. 1: 803. 1913.

Diphyes microdendron (Schltr.) Szlach. \& Rutk., comb. nov.

Basionym: Bulbophyllum microdendron Schltr., Repert. Spec. Nov. Regni Veg., Beih. 1: 821. 1913.

Diphyes montanum (Schltr.) Szlach. \& Rutk., comb. nov. Basionym: Bulbophyllum montanum Schltr., Repert. Spec. Nov. Regni Veg., Beih. 1: 801. 1913.

Diphyes myrtillus (Schltr.) Szlach. \& Rutk., comb. nov. Basionym: Bulbophyllum myrtillus Schltr., Repert. Spec. Nov. Regni Veg., Beih. 1: 821. 1913.

Diphyes navicula (Schltr.) Szlach. \& Rutk., comb. nov. Basionym: Bulbophyllum navicula Schltr., Repert. Spec. Nov. Regni Veg., Beih. 1: 819. 1913.
Diphyes nigrilabium (Schltr.) Szlach. \& Rutk., comb. nov. Basionym: Bulbophyllum nigrilabium Schltr., Repert. Spec. Nov. Regni Veg., Beih. 1: 800. 1913.

Diphyes oblanceolatum (Schltr.) Szlach. \& Rutk., comb. nov.

Basionym: Bulbophyllum oblanceolatum Schltr., Repert. Spec. Nov. Regni Veg., Beih. 1: 817, 1049. 1913.

Diphyes oligochaete (Schltr.) Szlach. \& Rutk., comb. nov. Basionym: Bulbophyllum oligochaete Schltr., Repert. Spec. Nov. Regni Veg., Beih. 1: 819. 1913.

Diphyes plumula (Schltr.) Szlach. \& Rutk., comb. nov. Basionym: Bulbophyllum plumula Schltr., Repert. Spec. Nov. Regni Veg., Beih. 1: 796. 1913.

Diphyes polyblepharon (Schltr.) Szlach. \& Rutk., comb. nov

Basionym: Bulbophyllum polyblepharon Schltr. in K.Schum. \& Lauterb., Nachtr. Fl. Deutsch. Sudsee: 214. 1905.

Diphyes ptilotes (Schltr.) Szlach. \& Rutk., comb. nov. Basionym: Bulbophyllum ptilotes Schltr., Repert. Spec. Nov. Regni Veg., Beih. 1: 797. 1913.

Diphyes punamense (Schltr.) Szlach. \& Rutk., comb. nov. Basionym: Bulbophyllum punamense Schltr., Repert. Spec. Nov. Regni Veg., Beih. 1: 797. 1913.

Diphyes purpurascens (Bail) Szlach. \& Rutk., comb. nov. Basionym: Bulbophyllum purpurascens Bail, Proc. Roy. Soc. Queensl. 1: 88. 1884, non Teijsm. \& Binn., Natuurk. Tijdschr. Ned. Indie 24: 308. 1862.

Diphyes quinquelobum (Schltr.) Szlach. \& Rutk., comb. nov.

Basionym: Bulbophyllum quinquelobum Schltr., Repert. Spec. Nov. Regni Veg., Beih. 1: 805. 1913.

Diphyes rarum (Schltr.) Szlach. \& Rutk., comb. nov. Basionym: Bulbophyllum rarum Schltr., Repert. Spec. Nov. Regni Veg., Beih. 1: 807. 1913.

Diphyes recurviflorum (J.J.Sm.) Szlach. \& Rutk., comb. nov.

Basionym: Bulbophyllum recurviflorum J.J.Sm., Ic. Bogor.: t. 118 B. 1903 .

Diphyes renipetalum (Schltr.) Szlach. \& Rutk., comb. nov. Basionym: Bulbophyllum renipetalum Schltr., Repert. Spec. Nov. Regni Veg., Beih. 1: 818. 1913.

Diphyes rhopaloblepharon (Schltr.) Szlach. \& Rutk., comb. nov.

Basionym: Bulbophyllum rhopaloblepharon Schltr., Repert. Spec. Nov. Regni Veg., Beih. 1: 811. 1913.

Diphyes scopula (Schltr.) Szlach. \& Rutk., comb. nov. Basionym: Bulbophyllum scopula Schltr., Repert. Spec. Nov. Regni Veg., Beih. 1: 805. 1913. 
Diphyes schillerianum (Rchb.f.) Szlach. \& Rutk., comb. nov.

Basionym: Bulbophyllum schillerianum Rchb.f. in Otto, Hamburg. Garten Blumenzeit. 16: 423. 1860.

Diphyes serpens (Schltr.) Szlach. \& Rutk., comb. nov.

Basionym: Bulbophyllum serpens Schltr., Repert. Spec. Nov. Regni Veg., Beih. 1: 804. 1913.

Diphyes serrulatum (Schltr.) Szlach. \& Rutk., comb. nov. Basionym: Bulbophyllum serrulatum Schltr. in K. Schum. \& Lauterb., Nachtr. Fl. Deutsch. Sudsee: 216. 1905.

Diphyes stenochilum (Schltr.) Szlach. \& Rutk., comb. nov. Basionym: Bulbophyllum stenochilum Schltr., Repert. Spec. Nov. Regni Veg., Beih. 1: 823. 1913.

Diphyes tenompokense (Schltr.) Szlach. \& Rutk., comb. nov.

Basionym: Bulbophyllum tenompokense J.J.Sm., Repert. Spec. Nov. Regni Veg. 36: 116. 1934.

Diphyes tentaculiferum (Schltr.) Szlach. \& Rutk., comb. nov.

Basionym: Bulbophyllum tentaculiferum Schltr., Repert. Spec. Nov. Regni Veg., Beih. 1: 782.1913.

Diphyes tortuosa Blume

Bijdr. Fl. Ned. Ind. 310: t. 311. 1825.

Diphyes triandrum (Schltr.) Szlach. \& Rutk., comb. nov. Basionym: Bulbophyllum triandrum Schltr., Repert. Spec. Nov. Regni Veg., Beih. 1: 810. 1913.

Diphyes unguilabium (Schltr.) Szlach. \& Rutk., comb. nov.

Basionym: Bulbophyllum unguilabium Schltr., Repert. Spec. Nov. Regni Veg., Beih. 1: 812. 1913.
Diphyes unicaudatum (Schltr.) Szlach. \& Rutk., comb. nov.

Basionym: Bulbophyllum unicaudatum Schltr., Repert. Spec. Nov. Regni Veg., Beih. 1: 816. 1913.

Diphyes vaccinioides (Schltr.) Szlach. \& Rutk., comb. nov. Basionym: Bulbophyllum vaccinioides Schltr., Repert. Spec. Nov. Regni Veg., Beih. 1: 820. 1913.

Diphyes verruculatum (Schltr.) Szlach. \& Rutk., comb. nov.

Basionym: Bulbophyllum verruculatum Schltr., Repert. Spec. Nov. Regni Veg., Beih. 1: 811. 1913.

Diphyes xanthotes (Schltr.) Szlach. \& Rutk., comb. nov. Basionym: Bulbophyllum xanthotes Schltr., Repert. Spec. Nov. Regni Veg., Beih. 1: 799. 1913.

\section{ACKNOWLEDGMENTS}

We are grateful to Barbara Jahn from the Library of the Botanical Museum and Botanical Garden in Berlin-Dahlem for her help in gathering original Blume's work.

\section{LITERATURE CITED}

BLUME C. L. 1825. Bijdragen tot de Flora van nederlandsch Indie. - Batavia: 310-319.

COMBER J. B. 1990. Orchids of Java. Bentham-Moxon Trust, Kew: 407 pp.

SEIDEFADEN G. 1979. Orchid Genera in Thailand. VIII. Bulbophyllum Thouars. - Dansk Bot. Arkiv 33(3): 1-228.

SEIDEFADEN G., WOOD J. J. 1992. The orchids of Pennisular Malaysia and Singapore. Olsen and Olsen, Fredensborg: 779 pp.

VERMEULEN J.J. 1991. Bulbophyllum. In: P.J. Cribb (ed.), Orchids of Borneo. 2: 1-304. Bentham-Moxon Trust, Kew and Toihaan Publ. Co, Kota Kinabalu. 